\title{
Matemática y Complejidad
}

\section{Mathematics And Complexity}

ID Luis Arturo Polanía-Quiza1; Daniel Enrique Ovalle-Cerquera²; Juan David Cárdenas-Lis ${ }^{3}$

\section{Resumen}

En este trabajo se presentan algunos elementos relacionados con el enfoque o filosofía de la complejidad y su integración con la matemática. Se describen las particularidades de los sistemas dinámicos nolineales como sistemas complejos. En este sentido, se hace énfasis en ver a las matemáticas como una disciplina unificadora de la ciencia: una disciplina transdisciplinar. Por otro lado, se sostiene que el Teorema de Incompletud de Kurt Gödel es una de las más significativas razones que sustenta la insuficiencia teórica de la lógica clásica a partir de fundamentos matemáticos. Lo anterior se ajusta a la noción de indecidibilidad de Murray Gell-Man, la cual introduce el tema de las lógicas no clásicas, en particular, la lógica del tercero incluido como la lógica de la complejidad.

Palabras Clave: Complejidad; Transdisciplina; Sistemas Dinámicos no-lineales; Lógica No-Clásica; Indecidibilidad; Sistemas Complejos

\begin{abstract}
In this paper are exposed some elements related to the approach or philosophy of the Complexity and it's integration with mathematics. Here are also presented particularities of the non-lineal dynamic systems as complex systems. In this way, Mathematics are seen as a, unifying discipline of science: an interdisciplinary discipline. On the other hand, Kurt Godels' incompleteness theorem was understood as one of the most significant reasons used to talk about the theoretical insufitiencies of classical logic, starting from mathematical fundaments, reaching the undecidability motion from Murray Gell-Man, which introduces to the non-classical logics, particularly the third included logic like the complexity logic.
\end{abstract}

Keywords: complexity; transdisciplinary; non-linear dynamic systems; non-classical logic; indecidibility; complex systems

Tipología: Artículo de revisión.

Recibido: 06/04/2018

Evaluado: $16 / 11 / 2018$

Aceptado: $19 / 12 / 2018$

Disponible en línea: 24/12/2018

Como citar este artículo: Polanía, A. Ovalle, D. Cárdenas, J. (2019) Matemática y Complejidad. Jangwa Pana, 18 (1) 120 131. Doi: http://dx.doi.org/10.21676/16574923.2693

\footnotetext{
${ }^{1}$ Colombiano. Lic. En Matemáticas y Física. MSc en Matemáticas. Docente Universidad Surcolombiana, Neiva, Huila, Colombia. lapola@usco.edu.co. Orcid ID: 0000-0002-4769-4455

${ }^{2}$ Colombiano. Matemático. danielovalle09@hotmail.com. Orcid ID: 0000-0001-5176-1916

${ }^{3}$ Colombiano. Matemático. juan_dacar31@hotmail.com. Orcid ID: 0000-0002-0013-7539
} 


\section{Introducción}

$\mathrm{E}$ 1 método tradicional de la ciencia implica un conjunto de conocimientos adquiridos de manera empírica y/o reflexiva por el ser humano. Esta ha evolucionado hasta el punto de crear y establecer técnicas o métodos globales que permiten la continuidad de un estudio formal, más avanzado y especializado del universo. A raíz de esto, se han generado varios tipos de ciencia: ciencias formales, ciencias de la naturaleza, ciencias sociales y humanas. Una de las ciencias más antiguas de la humanidad es la matemática: al tener como objeto de estudio entes abstractos (números, símbolos, figuras, etc.), se define como una ciencia formal. Gran parte de las ciencias de la naturaleza, como también de las ciencias sociales y humanas, ha hecho uso de las matemáticas para referenciar diferentes estudios, muy a groso modo: el cálculo y la geometría en las primeras, y la estadística en las dos últimas.

Desde los babilonios, egipcios y griegos de la Antiguiedad, hasta las civilizaciones de nuestro tiempo, las matemáticas han revolucionado el mundo. Gracias a la ciencia clásica, solidificada por el razonamiento científico (o Racionalismo: corriente filosófica emprendida por René Descartes durante la primera mitad del siglo XVII), la avanzada tecnología actual es fruto en gran parte de ella. No existe nada que no las toque. Pero es precisamente el avance de la ciencia, sujeta al método científico, la que la ha hecho limitada, insuficiente y carente de explicaciones del universo: en el mes de agosto del año 1900, David Hilbert (matemático alemán, 1862-1943) convoca el Segundo Congreso Internacional de Matemáticas, en el que plantea 23 problemas que resultaron claves para revolucionar a las matemáticas en los siguientes 100 años, y que pusieron en crisis la ciencia clásica. Algunos de los avances que se obtuvieron como inspiración de dicho congreso fueron: en primer lugar, desde la micro y macrofísica, la aparición de la mecánica cuántica (Max Planck) y la teoría de la relatividad (Albert
Einstein), lo que introdujo la complementariedad y la incertidumbre como principios bases de la "ciencia/física moderna"; en segundo lugar, se establecen como requisitos y problemas fundamentales de un sistema formal matemático, la consistencia y la completitud, de donde figuran como destacables los aportes de Kurt Gödel en la lógica (Teorema de incompletitud) y de Alan Turing en la computación (programas indecidibles, máquinas de Turing); en tercer lugar, el matemático francés Henri Poincaré descubrió que las ecuaciones diferenciales nolineales son las que mejores se adaptan al comportamiento de la dinámica cosmológica, lo que generó cualitativamente ciertos fenómenos en sus soluciones (Bifurcaciones y Caos); por último, Sierpinski, Koch y Peano aportaron la obtención de figuras extrañas totalmente incomprensibles desde la geometría euclidiana, lo que dio paso a la geometría fractal consolidada por el matemático polaco Benoit Mandelbrot. Luego, aproximadamente en el transcurso del tercio medio del siglo XX, la teoría de la información (Shannon y Weaver), la cibernética (Wiener y Stearns) y la teoría general de los sistemas de von Bertalanffy, enfatizan en el concepto de retroalimentación y una visión interdisciplinar en la ciencia.

Por último, a finales de los sesenta hasta la actualidad, emerge un gran número de teorías que busca explicar la variedad de fenómenos propios de la realidad y que hacen comprenderla: el pensamiento complejo (Edgar Morin), teoría de las estructuras disipativas (Ilya Prigogine), complejidad y simplicidad (Murray Gell-Mann), ciencias de la complejidad (Carlos Maldonado), son algunas de ellas. Términos como autoorganización, isomorfismo, analogía, transdisciplinariedad, recursividad, desorden, azar, entropía, adaptabilidad, entre otros, las fundamentan.

Desde finales del siglo XIX, la crisis del paradigma de la ciencia clásica es indiscutible, necesita un ingrediente que le permita estudiar, con algo más de razón, la realidad en que 
vivimos, sin parcelación, simplificación y reducción de lo existente: la complejidad converge con cada uno de los conceptos, nociones y teorías mencionadas anteriormente. Por tal motivo es necesaria una contextualización de lo que se entiende por "complejidad; y a partir de lo expuesto por algunos autores desde sus disciplinas, plantear la relación entre la matemática y las ciencias de la complejidad.

\section{Sobre la complejidad}

En principio hablar de pensamiento sistémico (von Bertalanffy, von Foester, G. Bateson, la escuela de Palo Alto, Humberto Maturana y Francisco Varela, entre otros), permitió hablar luego de complejidad, y se abordó desde dos vertientes principales: la primera, el pensamiento complejo, referenciada por la obra de Edgar Morin y sus seguidores (Carlos Delgado Diaz, Pedro L. Sotolongo, y otros); y la segunda, denominada ciencias de la complejidad, referenciada principalmente por los aportes de los miembros del Instituto Santa Fe. Dicho instituto fue fundado en 1984 en Nuevo México (Estados Unidos) dio origen a las ciencias de la complejidad, que se convirtió en su mayor exponente. Algunos de sus fundadores son: J. Doyne Farmer, Murray Gell-Mann, John H. Holland, Melanie Mitchell, Stuart Kauffman, Duncan Watts, etc.

En su artículo "Teorías de la complejidad y ciencias sociales. Nuevas Estrategias Epistemológicas y Metodológicas" Rodríguez y Aguirre (2011) se refieren a la complejidad como una perspectiva novedosa y marginal en la ciencia contemporánea; su carácter de novedad radica en que el estudio de la complejidad implica, en buena medida, un quiebre o discontinuidad en la historia de la ciencia o, más precisamente dicho, en la racionalidad científica occidental. La complejidad introduce, en el

4 Nos interesa que se entienda o se intuya algo sobre transdisciplina, pues hablar de pluri, multi, inter $\mathrm{y}$ transdisciplina, al igual que la Complejidad, es una discusión filosófica abordada por diversos autores que terreno de las ciencias, una racionalidad postclásica que habilita e incorpora problemas ignorados $\mathrm{o}$ vedados por el pensamiento científico moderno. Estos problemas involucran, en un sentido no exhaustivo, cuestiones relativas al desorden, el caos, la no-linealidad, el noequilibro, la indecidibilidad, la incertidumbre, la contradicción, el azar, la temporalidad, la emergencia, la auto-organización. La complejidad puede entenderse, por lo tanto, como un paradigma científico emergente que involucra un nuevo modo de hacer y entender la ciencia, extendiendo los límites y criterios de cientificidad, más allá de las fronteras de la ciencia moderna, ancladas sobre los principios rectores del mecanicismo, el reduccionismo y el determinismo (p.2).

Además, cuando Carlos Delgado Diaz y Pedro Luis Sotolongo escriben sobre "La complejidad y el diálogo transdisciplinario de saberes" (2006), exponen que la historia de la teoría o enfoque de la complejidad testifica que su corpus transdisciplinario (conceptual, metodológico y metódico) de conocimientos se ha ido constituyendo a partir del aporte de muchas disciplinas, entre las más relevantes: las exactas, naturales, sociales, técnicas y formales, que posteriormente las retroalimenta con lo elaborado transdisciplinariamente ${ }^{4}$. Por ello, definen Transdisciplina como:

el esfuerzo indagatorio que persigue obtener "cuotas de saber" análogas sobre diferentes objetos de estudio disciplinarios, multidisciplinarios o interdisciplinarios -incluso aparentemente muy alejados y divergentes entre síarticulándolas de manera que vayan conformando un corpus de conocimientos que trasciende cualquiera de dichas disciplinas, multidisciplinas e interdisciplinas (p.65).

convergen y divergen a la hora de dar sus aportes, y dicho debate no es uno de los fines a tratar en este escrito. 
De esta manera, algunos de los enfoques de la complejidad, como caos, atractores, espacio de fases, fueron y son citados por el estudio de la Dinámica Física; otras, como las ideas de estructuras disipativas y ciclos autocatalíticos, se implican a través de la termodinámica física, química y/o la químico-física; campos de estudio en comportamientos que limitan con el caos como las bifurcaciones y los fractales, que se desarrollan mediante la dinámica física y las matemáticas; y la de borrosidad o sets fuzzy se incorporaron desde la lógica y las ciencias sociales; por otro lado, el estudio de redes ya sean: red distribuida, red de redes, retroalimentaciones, conectividad, está dedicado a la cibernética, las neurociencias, la sociología (Delgado y Sotolongo, 2006).

Por lo tanto, al afectar el proceder científico, la complejidad se convierte en un tema-problema filosófico debatido por muchos autores que la entienden y la estudian de diversas maneras y cada uno llega a definirla según su propia filosofía. Algunas nociones de autores acerca de la complejidad son: tejido de constituyentes heterogéneas inseparablemente asociadas; presentan la paradoja de lo uno y lo múltiple (Morin, 1984), entendida como algo inmediatamente impredecible e incontrolable (Maldonado, 2007), aquellos fenómenos o problemas en donde intervienen un número amplio de factores o variables interrelacionados que conforman un todo orgánico (Weaver, 1948), es el resultado de atractores, rupturas de simetría o desequilibrios (Prigogine, 1997), complejidad bruta: longitud del mensaje más corto que describe un sistema, con una resolución dada, dirigido a un interlocutor distante y haciendo uso de un lenguaje y un conocimiento del mundo que ambas partes comparten (y saben que comparten) de antemano (Gell-Mann, 1995). Es relevante incluir estas definiciones en virtud de sus fundamentos, adicionalmente a las expuestas por M. Gell-Mann y Seth Lloyd en su artículo "Information measures, effective complexity, and total information. Complexity" donde citan 42 destacadas definiciones de complejidad, la mayor parte de ellas caracterizadas con principios que podemos relacionar con nociones matemáticas. Sin embargo, es importante saber que, por su naturaleza misma, la complejidad no busca dejar en el olvido a las disciplinas que hasta hoy existen; al contrario, busca complementarlas, establecer una conexión transversal entre ellas, para así dar soluciones correspondientes a problemas más ajustados a la realidad. Incluso, no existe una noción o definición única (universal) de complejidad, ya que, si existiera, no habría ningún tipo de sentido hablar de complejidad.

\section{Los sistemas dinámicos no-lineales como sistemas complejos}

Ordinariamente un sistema se conoce como un conjunto de elementos que se interrelacionan entre sí (Bertalanffy, 1928), dotado de una cierta organización. Existen muchas definiciones de sistema, por ejemplo, Edgar Morín lo define como la unidad global, compleja y organizada. Otras definiciones las cita Luz Arabany en su escrito "Teoría de Sistemas" (2002), donde sus autores correspondientes aportan que un sistema es:

Un conjunto de partes coordinadas y en interacción para alcanzar un objetivo (teleología); o una totalidad distinguible en un entorno $o$ ambiente en el cual interactúa, compuesta a su vez de elementos que interactúan también; o un grupo de componentes interrelacionados que trabajan en conjunto hacia una meta común mediante la aceptación de entradas $\mathrm{y}$ generando salidas en un proceso de transformación organizado; o es un conjunto de elementos dinámicamente relacionados entre sí, que realizan una actividad para alcanzar un objetivo, operando sobre entradas y proveyendo salidas procesadas. Se encuentra en un medio ambiente y constituye una totalidad diferente de otra (p.18-19). 
Ahora bien, para evitar caer en un simple juego de palabras a la hora de definir lo que es un sistema, consideramos que las definiciones anteriores exponen una idea clara de lo que significa, de donde podemos extraer dos características fundamentales: un sistema es dinámico y organizado. La naturaleza, la sociedad, los seres vivos, la economía mundial son sistemas dinámicos y organizados. La física, la biología, la sociología y otras disciplinas se dedican, en general, a estudiar y analizar sucesos o eventos que cambian con el tiempo y para ello utilizan ciertos elementos matemáticos que facilitan su labor: los sistemas dinámicos. Un sistema dinámico es un modelo matemático utilizado para estudiar procesos iterativos de naturaleza determinística o aleatoria. Todo proceso ocurre en su propio tiempo, y puede ser discreto o continuo. El proceso está caracterizado por variables y/o parámetros, las cuales interactúan mediante relaciones funcionales (lineales o no lineales)

Formalmente, un sistema dinámico es un par $(M, F)$ donde $M$ representa el espacio temporal, es decir, el ambiente del sistema, llamado espacio de estados o espacio de fases. $F$ es una aplicación del espacio de estados en él mismo $F: M \rightarrow M$ y se denomina ley de evolución del sistema o ley de transición de estados. El conjunto $M$ generalmente es una vecindad diferenciable.

Si el sistema dinámico es continuo, la aplicación $\mathrm{F}$ es un campo vectorial, definido por el lado derecho de un sistema de ecuaciones diferenciales:

$\frac{d x}{d t}=F(x), x \in M, t>0$

$\mathrm{Si}$ el sistema dinámico es discreto, $F$ generalmente es un difeomorfismo (una función diferenciable con inversa diferenciable), el sistema dinámico se expresa mediante una ecuación en diferencias:

$$
x_{n+1}=F\left(x_{n}\right), n=0,1,2, \ldots \text { con } x_{n} \in M
$$

Ahora bien, se hace innecesario desarrollar detalladamente el temario correspondiente a los sistemas dinámicos, pues lo primordial es identificar nociones de la complejidad en las matemáticas. Razón por la cual el enfoque prima hacia los sistemas dinámicos no lineales.

La no-linealidad, de hecho, es una característica fundamental de la complejidad. Autores como Carlos Delgado Diaz (2006) y Edgar Morin (1984) argumentan sobre ello. La realidad es de carácter no-lineal. En tanto se piense el tiempo como una línea dirigida de atrás hacia delante (pasado hacia el futuro), es complicado resaltar aquellas experiencias interiores, y que típicamente se cambian por simples ilusiones, disociaciones y rarezas, y su percepción, de cualquier modo, no tiene lugar en la naturaleza física y en la esencia del tiempo.

En "La interdisciplina desde la teoría de los sistemas complejos" (2005), Köppen, Mansilla y Miramontes intervienen sobre la no-linealidad de los sistemas complejos de la siguiente manera un sistema complejo consta de componentes individuales que interactúan $\mathrm{y}$, como producto de ello, pueden modificar sus estados internos. El número de componentes es suficientemente grande para que su estudio al estilo de Newton resolviendo una ecuación diferencial por cada grado de libertad- sea imposible, y suficientemente pequeño para que el formalismo de la mecánica estadística -donde promediar proporciona sentido al uso de variables macroscópicas- no sea válido. La interacción no es lineal $\mathrm{y}$, habitualmente, ésta resulta de dinámicas antagónicas. Un sistema complejo es reconocible por su comportamiento, en él suele haber auto-organización, frustración y evolución hacia la zona crítica, leyes de potencia espaciotemporales y, fundamentalmente, emergencia de patrones. (p.)

Además, manifiestan que se puede obtener un comportamiento macroscópico común en muchos sistemas, independientemente de sus composiciones microscópicas, entonces la descripción matemática más parsimoniosa del 
que sea el más sencillo de modelar, en principio, es válida para todos. De hecho, esta es la razón por la cual los modelos matemáticos existen, funcionan, y lo hacen muy bien; son el retrato de un arquetipo, de una clase de universalidad dinámica. Por consiguiente, no hay razón para extrañarse de que un modelo matemático originalmente formulado para algún problema de dinámica de poblaciones, sea también efectivo en epidemiología o para la propagación de rumores; tampoco que las herramientas de la mecánica estadística sean las mismas que las de la economía global, o que la conducta de agregados neuronales se parezca a la del comportamiento social de las especies gregarias de insectos (Köppen, Mansilla y Miramontes, 2005, p.10-11).

Es decir, las matemáticas facilitan la acción transdisciplinar en la ciencia. Cabe tener en cuenta la noción positivista, donde el conocimiento se basa en la observación y la experimentación, y cuyo recurso era la matemática. "Un mundo objetivo". (Najmanovich. 2001. p. 110). Por ejemplo, la teoría del caos, la teoría de catástrofes (René Thom) y la fractalidad permiten dicha transdisciplinariedad; a la final, son temas que se desarrollan a partir del estudio de los sistemas dinámicos no-lineales. Finalmente, "la discusión de inter, multi o transdisciplina pierde sentido, no sólo al ser derribados los muros entre disciplinas, sino por la emergencia de leyes y principios generales que se pueden estudiar cobijados bajo clases de universalidad dinámica" (Köppen, Mansilla y Miramontes, 2005, p.11). Estos principios organizativos actúan en el nivel mesoscópico: entre la dinámica microscópica y macroscópica; la capacidad de cómputo y la fractalidad son clases de universalidad dinámica.

De esta manera, la unificación de la ciencia se dará en el transcurso en que sea naturalmente posible reconocer tales clases y el metalenguaje al que apuntan quienes proponen la transdisciplina, y esta será, desde luego, la de la matemática, o en palabras más precisas, el principio unificador será la matemática de los sistemas no lineales (Köppen, Mansilla y Miramontes, 2005).

\section{Indecibilidad}

Existen variados comportamientos naturales que tienden a presentar estados intermedios, pero la lógica clásica, también conocida como lógica aristotélica o bivalente, se encarga de los fenómenos en que solo es concebible uno de los dos estados posibles, es decir, verdadero o falso, blanco o negro, cero o uno, encendido $\mathrm{o}$ apagado, etc.

El sistema lógico-formal tradicional soporta el sistema axiomático de las matemáticas. Si este sistema es completo, entonces cualquier cosa que sea verdadera es demostrable. Del mismo modo, cualquier falsedad es demostrablemente falsa (Castillo, 2005). Pero fue gracias a Kurt Gödel que se demostró que este sistema no era completo, y derrumbó así algunos sueños perseguidos por David Hilbert acerca de la consistencia de los números naturales. Esta búsqueda culminó con su teorema de incompletitud: cualquier sistema lógico deductivo consistente y suficientemente expresivo no es completo, tiene "lagunas" llenas de expresiones (proposiciones) que no pueden demostrarse si son verdaderas ofalsas, es decir, "habrá al menos una proposición que siendo verdadera no es demostrable" (Rodríguez, 2008, p.9).

Dicho teorema puso en jaque al sistema axiomático de las matemáticas, y abrió paso a las llamadas proposiciones indecidibles. La indecidibilidad es, entonces, una de las conclusiones fundamentales a las que llegó Gödel. Contemporáneamente a él, unos de los padres de la computación, Alan M. Turing, introducía dicho término en el contexto de la informática como el principio de indecidibilidad. El principio de indecidibilidad de Turing enuncia que no es posible escribir un programa que decida si otro programa cualquiera está correctamente escrito, en el sentido de nunca 
quedarse colgado (estancarse y quedarse dando vueltas sin avanzar). Es imposible construir una máquina que diga si un programa cualquiera se "colgará".

Por otro lado, Murray Gell-Mann en El Quark y el Jaguar, en lo referido a la "no computabilidad de la información algorítmica", señala que las proposiciones que son independientes de los axiomas, son las proposiciones indecidibles más celebradas. Murray toma como ejemplo la conjetura de Goldbach (Gell-Mann, 1995): el enunciado establece que todo número par mayor que 2, es el resultado de la suma de dos números primos, ya sean estos iguales o distintos. Teniendo en cuenta que un número primo es aquel que es mayor que 1 y que solo es divisible por la unidad y por él mismo. Es trivial empezar a verificar que la conjetura se va cumpliendo para números pares pequeños: $4=3+1, \quad 6=$ $3+3, \quad 8=5+3, \ldots, \quad 30=17+13=19+$ $11, \ldots, 100=97+3$, etcétera. Además, GellMann (1995) explica que: "con el uso de los ordenadores se ha podido verificar esta propiedad hasta números pares increíblemente grandes. Sin embargo, ningún ordenador puede demostrar la conjetura, que siempre podría dejar de ser cierta para algún número aún más grande" (p. 57). En otras palabras, esto significa que por ser todos los números pares mayores que 2 un conjunto infinito -pues es un conjunto que no es acotado, en este caso, superior- es imposible que un programa de ordenador (o algoritmo informático) pueda demostrarla. En consecuencia, sería una rigurosa demostración matemática la que podrá convertir esta conjetura en un verdadero teorema.

\section{Lógicas no-clásicas}

A partir de los años sesenta, surgen nuevos sistemas lógicos que no comparten algunos principios de la lógica clásica o simplemente incluyen un nuevo vocabulario, a los cuales se les denomina Lógicas No-Clásicas. Según Alba (2015), para el lógico español Alfredo Deaño), la lógica clásica no nos debe transportar a los griegos, sino más bien a considerar algunas de sus características fundamentales: Apofántica, Bivalente, Asertórica y Extensional. Apofántica quiere decir que tiene sentido preguntar por el valor de verdad de sus proposiciones. Bivalente, que toma en cuenta sólo dos valores de verdad: verdadero y falso. Asertórico, que no se admiten grados en los valores de verdad, es decir, no hay espacio para términos vinculados con alguna partícula de posibilidad o necesidad. Finalmente, la lógica clásica es extensional cuando toma como base el principio de sinonimia, es decir, si se sustituye un nombre por su sinónimo, en una proposición, no se altera el valor de verdad de la oración. Por otro lado, el aspecto en común que poseen las lógicas no clásicas es que todas rechazan algún principio que caracteriza a los sistemas de lógica clásica o bien incluyen nuevos teoremas con nuevos símbolos creados para expresarlos, lo que permite observarlas, a modo de especulación, como una extensión de la lógica clásica, por ejemplo, la lógica modal.

Para algunos el desarrollo de las lógicas no clásicas ha generado un desentendimiento respecto a la nueva postura de estas, es decir, ¿son las lógicas no clásicas una extensión o un rival de la lógica clásica? En cierta forma el adjetivo de "rival" asignado a estas lógicas no es más que un término histórico, pues la lógica clásica es la que se usaba antes. De igual manera, no se podría hablar de una extensión de ella, ya que se puede estudiar la lógica no clásica sin conocer la lógica clásica, así como también se puede estudiar geometría no euclidiana sin haber pasado por la euclidiana (simplemente es otra geometría).

En otras palabras, entender a la lógica no clásica como "rival" de la clásica, o como una crítica a ella, es un error. Por ejemplo, se cree que la lógica modal es una extensión de la lógica clásica, sin embargo, en la enseñanza de las lógicas, comúnmente se enseña primero la clásica para luego introducir, a modo de extensión, la modal. Pero se pudo haber dado primero esta "extensión", que entonces ya no sería una extensión, y sacar la lógica clásica como un caso particular de la lógica modal. De 
hecho, se puede tener sistemas matemáticos en los que se tienen diferentes tipos de números, por ejemplo, decir "diez más diez es cien" no implica que el sistema sea erróneo, lo mejor que se puede decir es que ya no se está ocupando el sistema decimal. De esta forma, la interpretación es lo que hace verla como extensión rival del sistema inicial.

Sin embargo, la conexión que hay entre las lógicas no-clásicas y la complejidad se da en el siguiente punto: vinculación de fenómenos y sistemas con comportamientos que tienden a un quiebre de estabilidad, es decir, presentan algunos puntos críticos, estados de transición y las transiciones de fases. Por tal motivo, en esta ciencia se articulan gran variedad de teorías (fluctuaciones, autoorganización, bifurcaciones, autocriticalidad organizada, y otras). He aquí algunas de las lógicas no-clásicas más representativas con sus respectivos autores: N. Da Costa, N. Belnap y Pinter (lógica paraconsistente), Ackermann, Anderson y Belnap (lógica de la relevancia), Prior (lógica temporal), Lukasiewics, Post, Kleene y Bochvar (lógicas polivalentes), Rescher y Zadeh (lógica difusa), Goldblatt (lógica cuántica) (Maldonado, 2007).

Si creemos que hay proposiciones que no son ni verdaderas ni falsas, sino todo lo contrario, como "la paradoja del mentiroso" (por ejemplo, a la proposición "esta oración es falsa" no es posible otorgarle un valor de verdad) o como algunas proposiciones matemáticas indemostrables, entonces se puede ir a una lógica polivalente o intuicionista. En suma, lógicas que rechacen el principio del tercio excluido.

\section{Lógica del tercero incluido: lógica de la complejidad}

Contraria sunt Complementa, afirmaba Niels Bohr.

"... es decir, día y noche, sol y luna, hombre y mujer, onda y partícula, razón y emoción, lógica e intuición, materia y espíritu, pragmatismo y misticismo, disciplina $y$ transdisciplina no como dicotomías, sino como complementos que tienden a fundirse y fusionarse, aún sin confundirse..."

(Manfred, 2004, p.14).

Es evidente que la rebelión que existe entre los mundos macrofísicos y microfísicos se debe a que son considerados contradictorios $y$ mutuamente excluyentes (A y no-A), aquellos mundos gobernados por paridades como ondapartícula, continuidad-discontinuidad, entre otros ejemplos.

La lógica usual (la clásica, vigente aún) se basa en tres axiomas o principios:

1. El axioma o principio de identidad: A es A.

2. El axioma o principio de la no contradicción: A no es no-A.

3. El axioma o principio del tercio excluido: No existe un tercer término $T$, que sea simultáneamente A y no-A.

Esta formulación lógica no permite dar solución a la paradoja planteada por la física cuántica, donde las partículas pueden ser vistas como onda y corpúsculo simultáneamente, ya que quebrantaría el segundo axioma o principio. Es complicado imaginar la idea de que exista un postulado que establezca un tercer término que sea verdadero y falso a la vez, (A y no-A). No obstante, al tomar como referente la idea planteada por Nicolescu, acerca de los niveles de realidad como "aquello que resiste nuestras experiencias, representaciones, descripciones, imágenes o formalizaciones matemáticas", la noción se vuelve un poco más permisible a reflexionar:

Imaginemos un triángulo en que uno de sus vértices está situado en un nivel de realidad, y los otros dos en otro nivel. Lo que en un nivel único aparecería como antagonismo entre dos elementos contradictorios (por ejemplo: onda A y partícula no-A), deja de serlo cuando un 
tercer elemento $\mathrm{T}$, ejercido desde otro nivel de realidad, torna lo aparentemente antagónico (onda y partícula) en una entidad unificada (quanton) percibida como no contradictoria (Manfred, 2004, p.15).

Ahora bien, la lógica de la complejidad es la lógica del tercero incluido, ya que por medio de la iteración permea distintas áreas del conocimiento de manera coherente, esto es, que a través de un proceso iterativo esta lógica permite describir la amistad entre niveles de realidad como sigue:

1. Un par de elementos contradictorios (A y no-A) situados en un determinado nivel de realidad es unificado por un estado $\mathrm{T}^{\prime}$ situado a un nivel contiguo de realidad;

2. A su vez, este estado T' está ligado a un par de elementos contradictorios ( $\mathrm{A}^{\prime}$ y no- $\mathrm{A}^{\prime}$ ) situados en su propio nivel;

3. El par contradictorio ( $\mathrm{A}^{\prime} \mathrm{y}$ no- $\left.\mathrm{A}^{\prime}\right)$ es, a su vez, unificado por un estado $T^{\prime \prime}$, situado a un distinto nivel de realidad, inmediatamente contiguo al cual cobijaba la terna ( $\mathrm{A}^{\prime}$, no- $\left.\mathrm{A}^{\prime}, \mathrm{y} \mathrm{T}^{\prime}\right)$. Tal proceso iterativo continúa indefinidamente hasta que todos los niveles de realidad posibles sean agotados (Manfred, 2004, p.16).

En el lenguaje matemático, este proceso iterativo sería:

$X \equiv$ Conjunto de estados unificados de los contradictorios.

$F \equiv$ Ley de transición de estados

$x_{0}=(A$, no $-A)$

$x_{1}=F\left(x_{0}\right)=\left(A^{\prime}\right.$, no $\left.-A^{\prime}, T\right)(3)$

$x_{2}=F\left(x_{1}\right)=\left(A^{\prime \prime}\right.$, no $\left.-A^{\prime \prime}, T^{\prime}\right)$

:

Este sistema dinámico discreto, no es más que un proceso de retroalimentación, es decir, el término siguiente está en función del término anterior. Y gracias a esta recursividad, se reinterpretan las metodologías de explicación lógica y su re-presentación, que junto a los avances de la física moderna (cuántica o de partículas), nace la necesidad de promover una lógica más incluyente dejando, parcialmente, el enfoque de excluyente del mundo. (Almarza, 2006, p.4).

La acción de esta lógica sobre los diferentes niveles de realidad, incita a crear una estructura abierta a la unidad de todos estos niveles, así, emergen consecuencias para la teoría del conocimiento, lo que implica la imposibilidad de construir una área completa cerrada en sí misma. Dentro del desarrollo de la física a inicios del siglo XX, con los aportes de Planck, Rutherford, Einstein, Bohr, De Broglie, Schrödinger, Heisenberg, Dirac se destacan tres movimientos conceptuales epistemológicos:

Concebir el átomo como un sistema formado por un núcleo con electrones girando a su alrededor (Rutherford Chadwick), la emergencia del carácter contradictorio de la materia, la partícula presentaba una dualidad, comportándose como onda o como corpúsculo, y el principio de incertidumbre (o indeterminación) de Heisenberg. (Rodríguez, 2010, p.79-80).

Como ejemplos donde se ilustra esta lógica encontramos las computadoras cuánticas, en donde el sistema hace uso de la mecánica cuántica. Aquí la idea que prima es la posibilidad de que una partícula se halle en dos lugares simultáneamente. El ordenador "clásico" maneja el "bit" como unidad de información, la cual solo puede tener un valor de 1 o 0 . El sistema equivalente para las computadoras cuánticas es el "qubits", lo que da la idea de la siguiente combinación: 00, 01, 10, 11 al mismo tiempo. La computadora binaria realiza cálculos de modo estándar donde los resultados son bivalentes -sí o no, encendido o apagado-, para el sistema cuántico es posible elaborar simultáneamente todos los cálculos y entregar la información de manera inmediata. Sin embargo, los estudiosos en esta área han tenido inconvenientes construyendo dispositivos con un número mayor 
de qubits, ya que sus ordenadores entran en la falencia llamada "decoherencia", es decir, cada bit cuántico pierde la propiedad de ser ambiguo, o lo que es lo mismo, se convierte en un simple bit clásico (BBC MUNDO, 2017).

Los principios en los que se basa la construcción de estos ordenadores cuánticos, se sitúan en la predicción del físico Paul Dirac (Como se citó en Nieves, 2017) que establece: "Todas y cada una de las partículas fundamentales del Universo tienen una "antipartícula", un gemelo idéntico a ellas, pero con carga eléctrica opuesta. Así, cuando una partícula se encuentra con su antipartícula, ambas se aniquilan, produciendo un breve destello de energía" (prr.1). Sin embargo, nueve años después, alrededor de 1937, el físico Ettore Majorana da un giro a la situación al predecir que de igual manera como existen las partículas conocidas como fermiones (algunas de ellas como el electrón, protón, neutrón, el neutrino y el quark), deberían existir aquellas que ellas mismas fueran su antipartícula.

Luego de ochenta años del enunciado de Majorana, algunos científicos de la Universidad de California en colaboración con la Universidad de Stanford, encontraron la primera evidencia de la existencia de los "fermiones de Majorana". Los experimentos realizados con materiales exóticos fueron dirigidos por los profesores de Jing Xia y Kang Wang, siguiendo los pasos del plan propuesto por Shoucheng Zhang. De esta manera, podrá darse un giro revolucionario no solo para los amantes de la informática, programación y tecnología, sino también para la ciencia, pues la capacidad de compilación y/o simulación aumenta, disminuyendo el costo computacional. "Zhang cree que en un futuro lejano los fermiones de Mejorana podrían usarse para construir robustos ordenadores cuánticos que no se vean afectados por el ruido ambiente, que es uno de los mayores obstáculos para su desarrollo" (Nieves, 2017, prr. 13).

\section{Conclusiones}

Los sistemas dinámicos no-lineales son los sistemas de la complejidad, en efecto, la nolinealidad junto a la recursividad son características indiscutibles de la complejidad, pues la interacción de sus componentes (o variables) es propensa a desarrollar algún tipo de bifurcación y, por ende, una catástrofe. Esta última es una ruta hacia el caos, también componente de la complejidad, por lo que se concluye que estos sistemas hacen parte de la matemática que hay en la complejidad. Por otra parte, los sistemas dinámicos discretos, haciendo uso de su retroalimentación o recursión, hacen de la matemática una ciencia transdisciplinar, pues es mediante la iteración que se trasciende en los niveles de realidad, argumentando a través de este proceso iterativo, la propuesta de una lógica para la complejidad.

Finalmente, al relacionar las matemáticas y la complejidad por medio de una elemental construcción epistemológica, se podrá hacer un estudio basado, por un lado, en las posibles aplicaciones de dicha relación, donde la teoría de grafos, los sistemas económicos, los sistemas sociales, las redes neurales, la evolución de poblaciones, la reproducción de especies, ofrecen un amplio campo de referencia aplicativo de los sistemas dinámicos no-lineales. Y por otro lado, profundizar, con una buena revisión y reflexión, la bibliografía destacada de algunas lógicas no-clásicas, ilustrándolas con ejemplos de diferentes disciplinas que hagan notar explícitamente el aporte de las matemáticas.

\section{Referencias}

Alba, A. (2015). Distinguir entre lógica clásica y no clásica. Justo en 1 click, (1). 
Almarza, F. (2006). Convergencia transdisciplinar: una nueva lógica de la Realidad. Tharsis. Universidad Central de Venezuela, Caracas.

BBC MUNDO. (2017). La Computadora cuántica, el "santo grial" de la informática, está a un paso de ser construida. http://www.bbc.com/mundo/noticias38856796

Castillo, C. (2005). Relatividad, incertidumbre, incompletitud e indecibilidad. Chile. http://chato.cl/blog/es/2005/08/relatividad _incertidumbre_incompletitud_e_indecidi bilidad.html

García, R. (2006). Sistemas Complejos. Barcelona, España: Gedisa Editorial.

Gell-Mann, M. (1995). El Quark y el Jaguar, Aventuras en lo simple y lo complejo. Barcelona, España: Tusquets.

Gell-Mann, M., Lloyd S. (1996). "Information measures, effective complexity, and total information”. Complexity, 2.

Köppen, E., Mansilla, R. y Miramontes, P. (2005). La interdisciplina desde la teoría de los sistemas complejos, Universidad Autónoma de México.

Maldonado, C. (1999). Esbozo de una filosofía de la lógica de la complejidad, Santa Fé de Bogotá. Visiones sobre la complejidad, $2^{\mathrm{a}}$ Edición, Colección "Filosofía y Ciencia" No. 1 ,

Maldonado C. (2007). El problema de una teoría general de la complejidad. Complejidad: ciencia, pensamiento y aplicaciones, Bogotá, Universidad Externado de Colombia.

Maldonado, C. (2009). La complejidad es un problema, no una cosmovisión, UCM Revista de Investigación, No 13. Bogotá.
Maldonado, C. (2017). Pensar. Lógicas no clásicas, Bogotá, Colombia: Universidad El Bosque, Facultad de Ciencias.

Maldonado, C. (2011). El Mundo de las Ciencias de la Complejidad: Una investigación sobre qué son, su desarrollo y sus posibilidades. Bogotá, Colombia.

Manfred, M. (2004). Fundamentos de la Transdisciplinaridad, Valdivia, Chile.

Montealegre Cárdenas, M, Londoño Betancourth, G. y Polanía Quiza, L. A. (2002). Fundamentos de los Sistemas Dinámicos: La interdisciplinariedad desde los Sistemas No lineales, Neiva, Huila: Universidad Surcolombiana.

Moriello, S. (s.f.) Dinámica de los Sistemas Complejos. Disponible en: http://www.pensamientocomplejo.org/doc s/files/Moriello_Dinamica\%20de\%20los $\%$ 20Sistemas\%20Complejos.pdf

Morin, E. (1984). Ciencia con Consciencia, Anthropos, Editorial del Hombre. Barcelona.

Morin, E. (1999). Introducción al pensamiento complejo, Buenos Aires: Gedisa.

Najmanovich, D. (2001). "Pensar la subjetividad. Complejidad, vínculos $\mathrm{y}$ emergencia”. Utopía y Praxis Latinoamericana.

Nieves, J. (2017). Logran, por primera vez, hallar un fermión de Majorana, la partícula que es su propia antipartícula. Madrid. Recuperado de: http://www.abc.es/ciencia/abci-logranprimera-hallar-fermion-majoranaparticula-propia-antiparticula201707210842_noticia.html

Nicolescu, B. (1994). La Transdisciplinariedad. Manifesto, Paris: Ediciones Du Rocher. 
Nicolescu, B. (1999). "Stephane Lupasco. L'homme et l'œuvre", Transdisciplinarité. La Rocher, París.

Prigogine, I. (1997). ¿Tan sólo una ilusión? Una exploración del orden al caos, Barcelona, España: Tusquets, Editores.

Ramirez, Luz. (2002). Teoría de Sistemas. Manizales, Colombia. Universidad Nacional de Colombia, Sede Manizales.

Rodriguez, L. (2008). Complejidad: la emergencia de nuevos valores epistémicos y no epistémicos en la historia de la ciencia contemporánea. I Encuentro Latinoamericano de Metodologías de las Ciencias Sociales. La Plata, Argentina.
Rodriguez, L. (2010). Contribuciones de la Historia de la Ciencia Contemporánea a la Emergencia del Paradigma de la Complejidad. Revista Hologramatica. VII, (13).

Rodríguez, L. y Aguirre, J. (2011). "Teorías de la Complejidad y Ciencias Sociales. Nuevas Estrategias Epistemológicas y Metodológicas", Nómadas. Revista Crítica de Ciencias Sociales y Jurídicas. Madrid, España.

Sotolongo Codina, P. L. y Delgado Díaz, C. J. (2006). Capítulo IV. La complejidad y el diálogo transdisciplinario de saberes, CLACSO, Consejo Latinoamericano de Ciencias Sociales.

Weaver, W. (1948). Science and Complexity, New York: Rockefeller Foundation, American Scientist. 